\title{
Is undernutrition risk associated with an adverse clinical outcome in spinal cord injured patients with severe pressure ulcer?
}

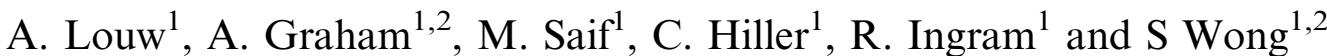 \\ ${ }^{1}$ National Spinal Injuries Centre, Stoke Mandeville Hospital, Aylesbury and ${ }^{2}$ Health Service Research, City University \\ London
}

Pressure ulcers (PU) are serious, costly and potentially life-long complications of spinal cord injury (SCI). Co-morbid conditions such as reduced mobility, sensory impairment and old age have been identified as risk factors for PU but others such as nutrition-risk is debated $^{1}$. The objective of this study is to (1) establish the incidence and location of grade 3 or above PU; (2) describe the prevalence of undernutrition risk using a validated disease-specific nutrition screening tool - Spinal Nutrition Screening Tool (SNST) ${ }^{2}$; (3) evaluate whether under-nutrition risk is associated with a worse clinical outcome in respect of length of in-patient hospital stay (LOS); bed-rest days and mortality within 12 months of admission to SCI centre. A retrospective review of patients admitted for PU management from January 2012 to December 2013 at the National Spinal Injuries Centre, Stoke Mandeville, UK was completed. Data collected included PU, medical and nutrition history as well as LOS, duration of bed-rest and mortality within 12 months of admission. A total of 779 patients were admitted during January 2012 to December 2013, of which 66 (8.5\%) patients (mean age: 61 years; $24.3 \%$ female) with SCI (24.2\% tetraplegia; $83.3 \%$ complete SCI) were admitted for PU management with a grade 3 or above PU. The top 3 sites for PU were Ischium (58.6\%); Sacral (21.5\%) and Trochanter $(12.3 \%)$. None of these patients had trace-elements checked. Only $23(34.8 \%)$ of patients were referred for nutrition assessment/intervention. These patients tends to have multiple PU $(52.1 \%$ v $20.9 \%, p<0.001)$ and lower serum-albumin $(\mathrm{g} / \mathrm{L}: 25 \mathrm{v} 30, \mathrm{p}=0.009)$. The patients initially undernourished or at-risk of undernutrition $(56.1 \%)$ had a significantly higher 12 -month mortality ( $\%$ deceased: $27.8 \%$ v $6 \cdot 7 \%$, p $=0.026)$. Non-significant differences were observed for LOS (undernutrition risk: $116 \mathrm{v} 78.5 \mathrm{~d}, \mathrm{p}=0.458$ ) and bed rest days (undernutrition risk: $74.5 \mathrm{~d}$ $\mathrm{v} 58.5 \mathrm{~d}, \mathrm{p}=0.456$ ). Many factors contribute in the development, course and treatment of PU. The present study suggests that undernutrition risk, as identified by the SNST, is associated with adverse clinical outcome. Routine nutrition screening should be helpful in improving clinical outcomes if it promotes more appropriate and effective nutrition intervention. Further study should address whether nutrition intervention is cost- and clinical- effective.

1. National Institute for Health and Care Excellence (2014) Pressure ulcers: prevention and management of pressure ulcers. NICE, London. http://bit. ly/1GkYJra

2. Wong et al. (2012) Eur J Clin Nutr 66, 382-387. 